\title{
Parameters monitoring system for the experimental setup SCAN-3
}

\author{
Dmitriy Sakulin ${ }^{1, \star}$ \\ ${ }^{1}$ Joint Institute for Nuclear Research, 141980 Dubna, Moscow region, Russia
}

\begin{abstract}
This overview begins with the presentation of General ideas about the physical parameters of SCAN-3. In addition, the basic principles of the monitoring system. The configuration of the web server of the monitoring system. In conclusion, the testing of the monitoring system on modules E-cal for the spectrometer BM@N.
\end{abstract}

\section{Introduction}

To perform the modern research is unthinkable without the use of computers. It is an important part of communication between the detectors and researchers. The development of computer technology and communications have allowed direct participants not to remain in the experiment, but to be active participants of these studies. This ideology is central in the modern experiment. System with this ideology will be presented in this overview.

\section{The model of the monitoring system}

\subsection{Experimental setup SCAN-3}

SCAN-3 is a three-arm Magnetic Spectrometer on the Nuclotron internal target for precision measurements of charged particles and neutrons. [1] It includes the following elements shown in figure 1. Required parameters of SCAN-3:

- Achieving the required precision is not possible without correction setup systems;

- The required accuracy of registration of the particle momentum is 1-2 percent;

- The accuracy of measurement depends on external parameters.

^e-mail: facolco@gmail.com 


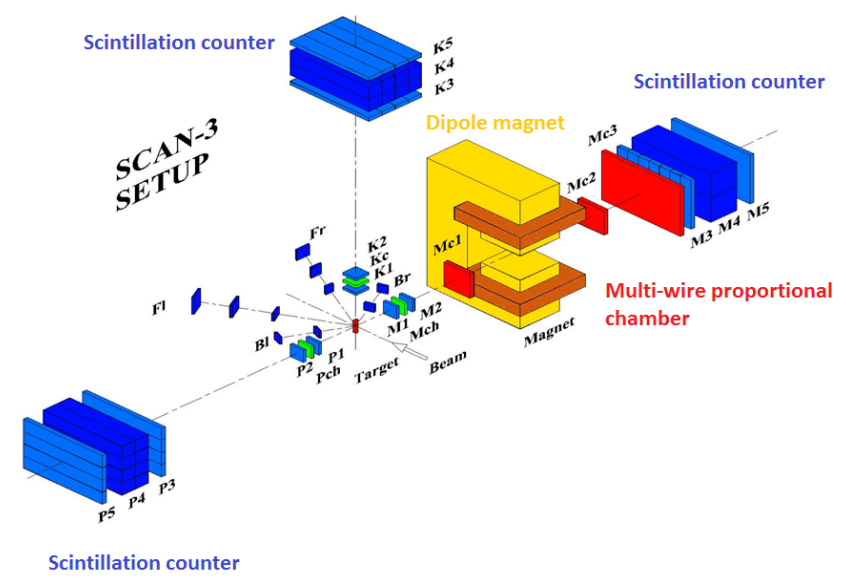

Figure 1. Experimental setup of SCAN-3.

\subsection{Main tasks of the monitoring system}

The considered system provides an opportunity:

- Collect, process, present and visualize data;

- Multisensory processing;

- Remote monitoring and control for the processes in the setup;

- Calibration and testing of individual subsystems.

Also, this work can be used for educational purposes.

\subsection{Basic principles of the monitoring system}

- Sensors are placed directly on the elements of the experiment;

- Using hardware and software for collection data (Arduino UNO platform);

- Using open-source relational database management system for storage data (MySQL);

- It is possible to use "raw" data;

- User-friendly interface through Highstock.

Arduino is an open-source electronics platform based on easy-to-use hardware and software. It is intended for anyone making interactive projects.

\subsection{Block diagram of the monitoring system}

Data from the sensors are transmitted to the database through internet connection (figure 2). The local server is located remotely. The clients access the server for the necessary information. 


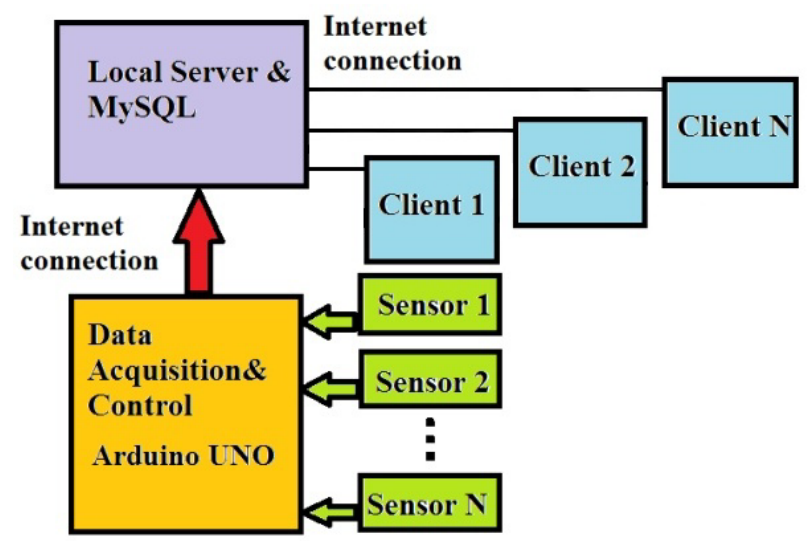

Figure 2. Block diagram of the monitoring system.

\subsection{Realization of the system}

[2]Arduino module with connected sensors looks as follows (figure 3). And it has also two sensors humidity and magnetic field.

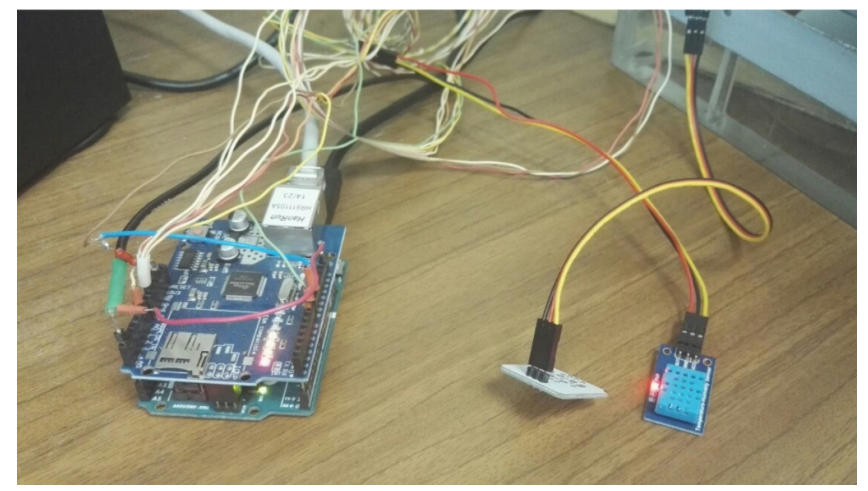

Figure 3. Arduino module with connected sensors.

\section{Application of the monitoring system during testing of ECAL module for BMaN spectrometer}

This monitoring system has been tested with ECAL module for BMaN spectrometer. In the test we considered an electromagnetic calorimeter designed to measure energy of particles which interact with matter primarily through the electromagnetic interaction (photons, charged leptons). The photos show the location of the sensors (figure 4). 


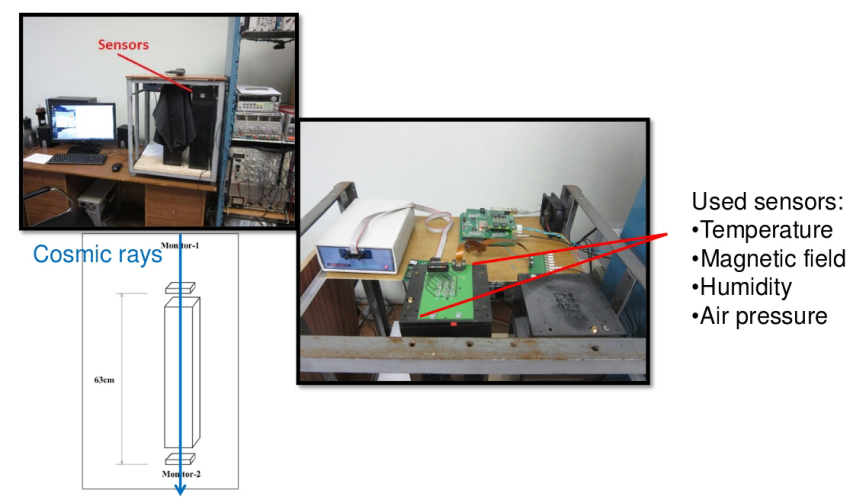

Figure 4. E-cal modules.

\subsection{Testing monitoring of triggers}

[3]The graph shows the monitoring of triggers from cosmic rays (figure 5). All days of experiment are accumulated and visualized in one day. Highstock in pure JavaScript, including sophisticated navigation options like a small navigator series, preset date ranges, date picker, scrolling and panning.

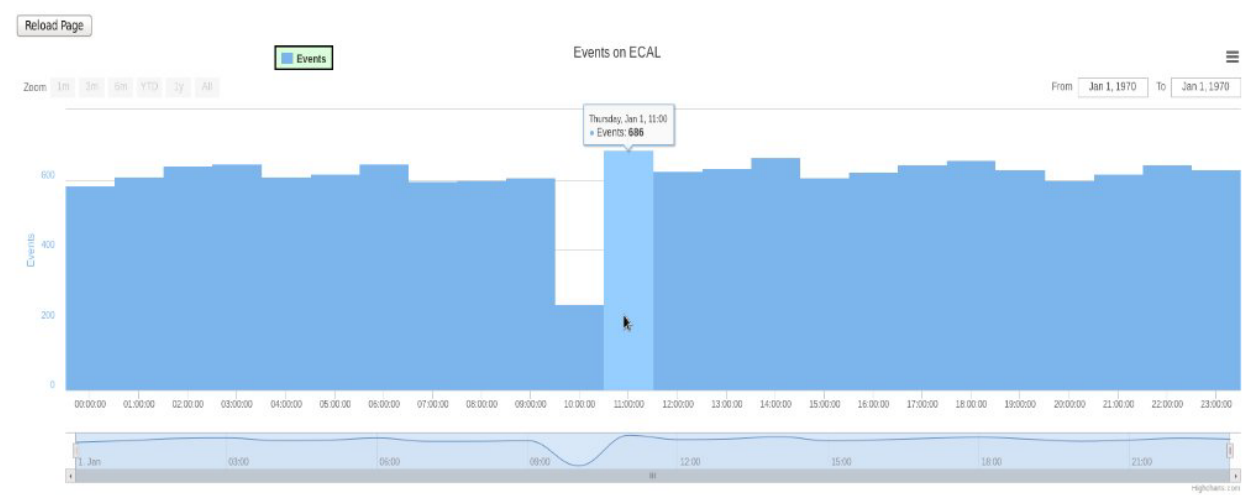

Figure 5. Triggers.

\subsection{Visualization of data}

This graph visually shows the values of the measuring sensors (figure 6). Highstock runs on the client side and it does not matter how the server is configured. This means that if the server is running PHP and MySQL or any other type of server in conjunction with the SQL, you can dynamically create the HTML and JavaScript with required Highstock. 


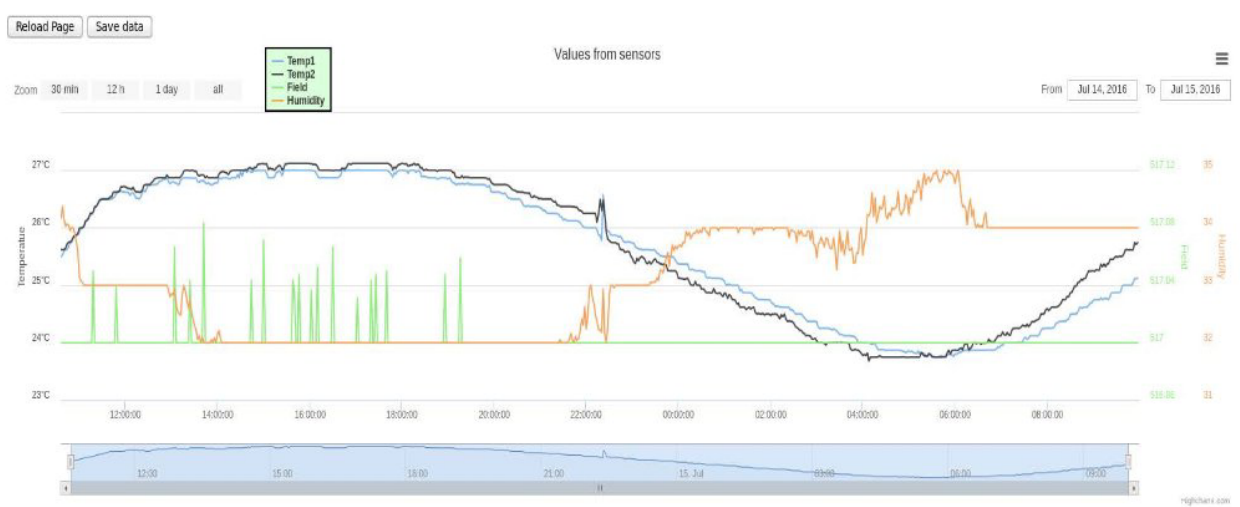

Figure 6. Visualization of data from sensors.

\subsection{Temperature dependence}

Significant changes in the readings of the magnetic field and the humidity are not detected. Therefore, we considered the temperature only. The graphs show the temperature dependence of the amplitudes of the signals (figure 7). The dependence for each channel was different. So after a temperature compensation for each channel we obtained the following results.

Temperature dependence of the amplitude of the signal.

Thermal compensation is turned OFF. Module N17 7 Jul 2016.

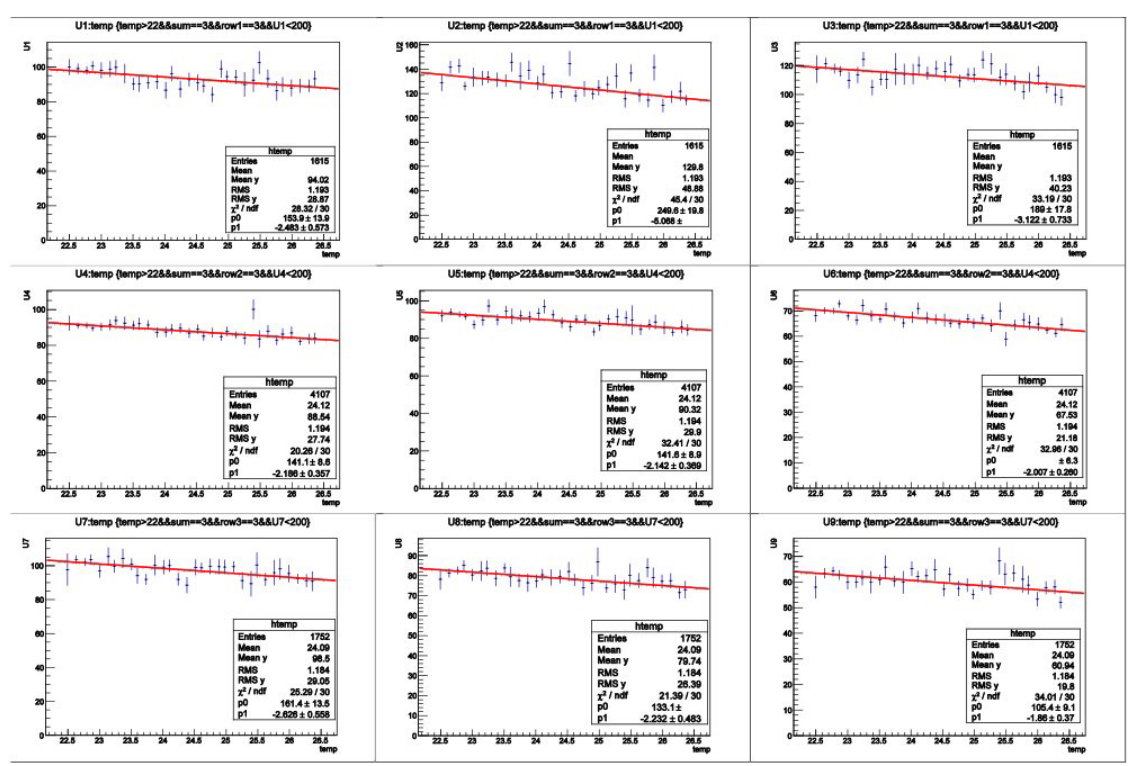

Figure 7. Temperature dependence. 


\section{Results}

The graph shows the amplitudes of the 9 ADC channels (figure 8). The top graph without temperature compensation depicts the variation of the amplitude of the signal. The variation of signal disappears after the temperature compensation. That allows to achieve the required accuracy.
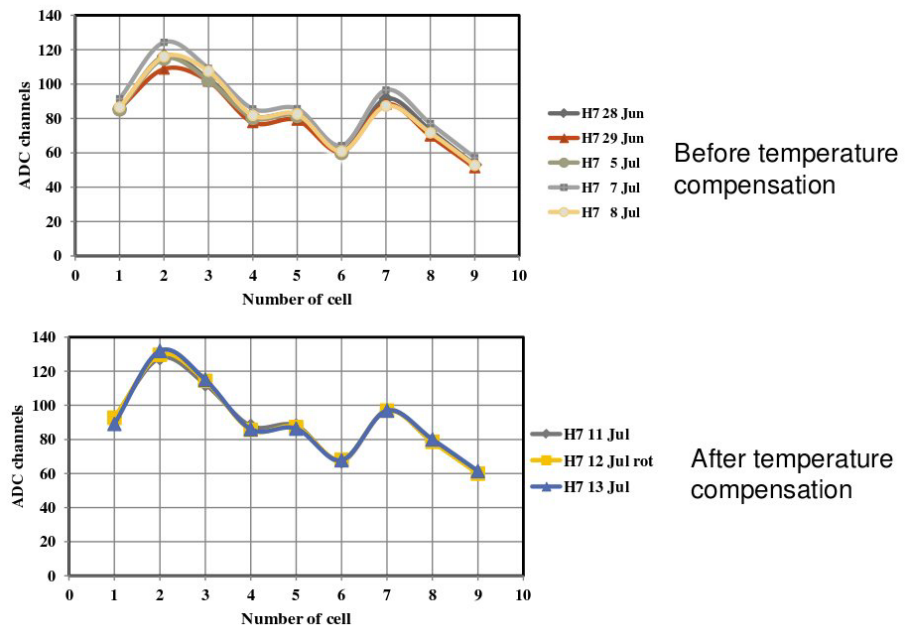

After temperature compensation

Figure 8. Graph measuring the amplitudes.

\section{Conclusion}

This monitoring system will be used in the system of slow control in the installation of the SCAN-3 on the Nuclotron, and also in the BM@N project. The system can be modified for any setups.

\section{References}

[1] New status of the project "n-nuclei" at the NUCLOTRON (2015), PoS (Baldin ISHEPP XXII) 125

[2] https://www.arduino.cc/

[3] http://www.highcharts.com/products/highstock 